\title{
WEYL'S THEOREM, $a$-WEYL'S THEOREM, AND LOCAL SPECTRAL THEORY
}

\author{
RAÚL E. CURTO AND YOUNG MIN HAN
}

\begin{abstract}
We give necessary and sufficient conditions for a Banach space operator with the single valued extension property (SVEP) to satisfy Weyl's theorem and $a$-Weyl's theorem. We show that if $T$ or $T^{*}$ has SVEP and $T$ is transaloid, then Weyl's theorem holds for $f(T)$ for every $f \in H(\sigma(T))$. When $T^{*}$ has SVEP, $T$ is transaloid and $T$ is $a$-isoloid, then $a$-Weyl's theorem holds for $f(T)$ for every $f \in H(\sigma(T))$. We also prove that if $T$ or $T^{*}$ has SVEP, then the spectral mapping theorem holds for the Weyl spectrum and for the essential approximate point spectrum.
\end{abstract}

\section{IntRoduction}

Let $B(X)$ denote the algebra of bounded linear operators acting on an infinite dimensional complex Banach space $X$. If $T \in B(X)$ we shall write $N(T)$ and $R(T)$ for the null space and range of $T$, respectively.

1991 Mathematics Subject Classification. Primary 47A10, 47A53, 47A11; Secondary 47A15, 47B20.

Key words and phrases. Weyl's theorem, Browder's theorem, $a$-Weyl's theorem, $a$-Browder's theorem, single valued extension property.

The research of the first named author was partially supported by NSF grants DMS-9800931 and DMS-0099357. 
Also, let $\alpha(T):=\operatorname{dim} N(T), \beta(T):=\operatorname{dim} X / R(T)$, and let $\sigma(T)$ denote the spectrum of $T, \pi_{0}(T)$ the eigenvalues of $T$, and $\pi_{0 f}(T)$ the eigenvalues of finite multiplicity of $T$. An operator $T \in B(X)$ is called Fredholm if it has closed range, finite dimensional null space, and its range has finite co-dimension. The index of a Fredholm operator $T$ is given by

$$
i(T):=\alpha(T)-\beta(T) .
$$

An operator $T \in B(X)$ is called Weyl if it is Fredholm of index zero, and Browder if it is Fredholm "of finite ascent and descent;" equivalently ([11, Theorem 7.9.3]) if $T$ is Fredholm and $T-\lambda$ is invertible for sufficiently small $\lambda \neq 0$ in $\mathbb{C}$. The essential spectrum $\sigma_{e}(T)$, the Weyl spectrum $\omega(T)$, and the Browder spectrum $\sigma_{b}(T)$ of $T \in B(X)$ are defined by ([10], [11])

$$
\begin{gathered}
\sigma_{e}(T):=\{\lambda \in \mathbb{C}: T-\lambda \text { is not Fredholm }\}, \\
\omega(T):=\{\lambda \in \mathbb{C}: T-\lambda \text { is not Weyl }\},
\end{gathered}
$$

and

$$
\sigma_{b}(T):=\{\lambda \in \mathbb{C}: T-\lambda \text { is not Browder }\}
$$

respectively. Evidently

$$
\sigma_{e}(T) \subseteq \omega(T) \subseteq \sigma_{b}(T)=\sigma_{e}(T) \cup \operatorname{acc} \sigma(T)
$$

where we write acc $K$ for the accumulation points of $K \subseteq \mathbb{C}$. 
If we write iso $K:=K \backslash$ acc $K$ then we let

$$
\pi_{00}(T):=\{\lambda \in \text { iso } \sigma(T): 0<\alpha(T-\lambda)<\infty\}
$$

denote the set of isolated eigenvalues of finite multiplicity, and we let

$$
p_{00}(T):=\sigma(T) \backslash \sigma_{b}(T)
$$

denote the set of Riesz points of $T$.

We say that Weyl's theorem holds for $T \in B(X)$ if

$$
\sigma(T) \backslash \omega(T)=\pi_{00}(T)
$$

and that Browder's theorem holds for $T \in B(X)$ if

$$
\sigma(T) \backslash \omega(T)=p_{00}(T)
$$

In [21], H. Weyl proved that (1.1) holds for hermitian operators. Weyl's theorem has been extended from hermitian operators to hyponormal operators, to Toeplitz operators «, and to several classes of operators including seminormal operators ([2], [3]).

In this article we give necessary and sufficient conditions for a Banach space operator with the single valued extension property (SVEP) to satisfy Weyl's theorem (Theorem 2.2) and $a$-Weyl's theorem (Corollary 3.3). (For the relevant definitions please see below.) We show that if $T$ or $T^{*}$ has SVEP and $T$ is transaloid, then Weyl's theorem holds for $f(T)$ for every $f \in H(\sigma(T))$ (Theorems 2.5 and 3.5). We establish that if $T^{*}$ has SVEP, and if $T$ is transaloid and $a$-isoloid, then $a$-Weyl's theorem holds for $f(T)$ for every $f \in H(\sigma(T))$ (Theorem 3.5). We also prove that 
if $T$ or $T^{*}$ has SVEP, then the spectral mapping theorem holds for the Weyl spectrum (Corollary 2.6) and for the essential approximate point spectrum (Theorem 3.1).

To describe some of the above mentioned results, we consider the sets

$$
\begin{aligned}
& \Phi_{+}(X)=\{T \in B(X): R(T) \text { is closed and } \alpha(T)<\infty\}, \\
& \Phi_{-}(X)=\{T \in B(X): R(T) \text { is closed and } \beta(T)<\infty\},
\end{aligned}
$$

and

$$
\Phi_{+}^{-}(X)=\left\{T \in B(X): T \in \Phi_{+}(X) \text { and } i(T) \leq 0\right\} .
$$

Moreover,

$$
\sigma_{l e}(T):=\left\{\lambda \in \mathbb{C}: T-\lambda \notin \Phi_{+}(X)\right\}
$$

is the left essential spectrum,

$$
\sigma_{r e}(T):=\left\{\lambda \in \mathbb{C}: T-\lambda \notin \Phi_{-}(X)\right\}
$$

is the right essential spectrum,

$$
\sigma_{e a}(T):=\left\{\lambda \in \mathbb{C}: T-\lambda \notin \Phi_{+}^{-}(X)\right\}
$$

is the essential approximate point spectrum, and

$$
\sigma_{a}(T):=\{\lambda \in \mathbb{C}: R(T-\lambda) \text { is not closed or } \alpha(T-\lambda)>0\}
$$

is the approximate point spectrum. We now let

$$
\pi_{00}^{a}(T):=\left\{\lambda \in \text { iso } \sigma_{a}(T): 0<\alpha(T-\lambda)<\infty\right\},
$$


denote the set of eigenvalues of finite multiplicity which are isolated in $\sigma_{a}(T)$, and we let

$$
\sigma_{a b}(T):=\bigcap\left\{\sigma_{a}(T+K): T K=K T \text { and } K \in K(X)\right\}
$$

denote the Browder essential approximate point spectrum, where $K(X)$ is the set of all compact operators on $X$. Observe that $\sigma_{e a}(T) \subseteq \sigma_{a b}(T)$ for every $T \in B(X)$.

We say that $a$-Weyl's theorem holds for $T \in B(X)$ if

$$
\sigma_{a}(T) \backslash \sigma_{e a}(T)=\pi_{00}^{a}(T)
$$

and that $a$-Browder's theorem holds for $T \in B(X)$ if

$$
\sigma_{e a}(T)=\sigma_{a b}(T)
$$

It is well known ([0], [12], [19]) that if $T \in B(X)$ then we have:

$a$-Weyl's theorem $\Longrightarrow$ Weyl's theorem $\Longrightarrow$ Browder's theorem;

$a$-Weyl's theorem $\Longrightarrow a$-Browder's theorem $\Longrightarrow$ Browder's theorem.

V. Rakočević [19 has shown that (1.3) holds for cohyponormal operators. More recently, S.V. Djordjević and D.S. Djordjević [6] have shown that if $T^{*}$ is quasihyponormal then $a$-Weyl's theorem holds for $T$.

An operator $T \in B(X)$ is called isoloid if every isolated point of $\sigma(T)$ is an eigenvalue of $T$. If $T \in B(X)$, we write $r(T)$ for the spectral radius of $T$; it is well known that $r(T) \leq\|T\|$. An operator $T \in B(X)$ is called normaloid if $r(T)=\|T\| . X \in B(X)$ is called a quasiaffinity if it has 
trivial kernel and dense range. $S \in B(X)$ is said to be a quasiaffine transform of $T \in B(X)$ (notation: $S \prec T$ ) if there is a quasiaffinity $X \in B(X)$ such that $X S=T X$. If both $S \prec T$ and $T \prec S$, then we say that $S$ and $T$ are quasisimilar.

We say that $T \in B(X)$ has the single valued extension property (SVEP) if for every open set $U$ of $\mathbb{C}$ the only analytic solution $f: U \longrightarrow X$ of the equation

$$
(T-\lambda) f(\lambda)=0 \quad(\lambda \in U)
$$

is the zero function ([5], [17]). Given an arbitrary operator $T \in B(X)$, the local resolvent set $\rho_{T}(x)$ of $T$ at the point $x \in X$ is defined as the union of all open subsets $U$ of $\mathbb{C}$ for which there is an analytic function $f: U \longrightarrow X$ which satisfies

$$
(T-\lambda) f(\lambda)=x \quad(\text { all } \lambda \in U)
$$

The local spectrum $\sigma_{T}(x)$ of $T$ at $x$ is then defined as

$$
\sigma_{T}(x):=\mathbb{C} \backslash \rho_{T}(x)
$$

For an arbitrary operator $T \in B(X)$, we define the local (resp. glocal) spectral subspaces of $T$ as follows. Given a set $F \subseteq \mathbb{C}$ (resp. a closed set $G \subseteq \mathbb{C}$ ),

$$
X_{T}(F):=\left\{x \in X: \sigma_{T}(x) \subseteq F\right\}
$$


(resp.

$$
\begin{aligned}
\mathcal{X}_{T}(G) & :=\{x \in X: \text { there exists an analytic function } \\
& f: \mathbb{C} \backslash G \rightarrow X \text { that satisfies }(T-\lambda) f(\lambda)=x \text { for all } \lambda \in \mathbb{C} \backslash G\}) .
\end{aligned}
$$

An operator $T \in B(X)$ has Dunford's property (C) if the local spectral subspace $X_{T}(F)$ is closed for every closed set $F \subseteq \mathbb{C}$; such operators automatically have SVEP [17].

We will use often the following two results.

Lemma 1.1. (迎, Theorem 2.6]) Let $T \in B(X)$ be an operator satisfying $S V E P$, and let $\lambda \notin \sigma_{e}(T)$. Then $T-\lambda$ has SVEP if and only if $T-\lambda$ has finite ascent.

Lemma 1.2. (14, Theorem 3.1]) Let $T \in B(X)$ and assume that $\lambda \in$ $\pi_{00}(T)$. Then $T=T_{1} \oplus T_{2}$ with respect to the decomposition $X=$ $\mathcal{X}_{T}(\{\lambda\}) \oplus K(T-\lambda)$, and $\sigma\left(T_{1}\right)=\{\lambda\}, \sigma\left(T_{2}\right)=\sigma(T) \backslash\{\lambda\}$, where $K(T):=\left\{x \in X: T x_{n+1}=x_{n}, T x_{1}=x,\left\|x_{n}\right\| \leq c^{n}\|x\|(n=\right.$ $1,2, \ldots)$ for some $\left.c>0, x_{n} \in X\right\}$.

\section{Extensions of Weyl's theorem}

The following theorem relates Weyl's theorem to local spectral theory. As motivation for the proof, we use some ideas in [16] and [17].

Theorem 2.1. Let $T \in B(X)$ and assume that $\mathcal{X}_{T}(\{\lambda\})$ is finite dimensional for each $\lambda \in \pi_{0 f}(T)$. Then Weyl's theorem holds for $T$. 
Proof. We must show that $\sigma(T) \backslash \omega(T)=\pi_{00}(T)$. Suppose that $\lambda \in$ $\sigma(T) \backslash \omega(T)$. Then $T-\lambda$ is Weyl but not invertible. Then $\lambda \in \pi_{0 f}(T)$, and hence $\operatorname{dim} \mathcal{X}_{T}(\{\lambda\})<\infty$. Since $\mathcal{X}_{T}(\{\lambda\})$ is an invariant subspace for $T$, we can write

$$
T=\left(\begin{array}{ll}
A & C \\
0 & B
\end{array}\right),
$$

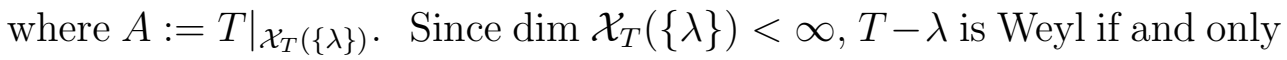
if $B-\lambda$ is Weyl. Thus, $B-\lambda$ is Weyl. However, $N(T-\lambda) \subseteq \mathcal{X}_{T}(\{\lambda\})$, hence $B-\lambda$ is injective. Therefore $B-\lambda$ is invertible. Recall now that since $\operatorname{dim} \mathcal{X}_{T}(\{\lambda\})<\infty, \sigma(A)$ must be finite and $\sigma(T)=\sigma(A) \bigcup \sigma(B)$. Thus, $\lambda$ is an isolated point of $\sigma(T)$. Therefore $\lambda \in$ iso $\sigma(T) \backslash \omega(T)$, and by the punctured neighborhood theorem, $\lambda \in \pi_{00}(T)$.

Conversely, suppose that $\lambda \in \pi_{00}(T)$. By Lemma 1.2, $T=T_{1} \oplus T_{2}$ on $\mathcal{X}_{T}(\{\lambda\}) \oplus K(T-\lambda)$, with $\sigma\left(T_{1}\right)=\{\lambda\}$ and $\sigma\left(T_{2}\right)=\sigma(T) \backslash\{\lambda\}$. Since $\operatorname{dim} \mathcal{X}_{T}(\{\lambda\})<\infty, T-\lambda$ is Weyl. Therefore $\lambda \in \sigma(T) \backslash \omega(T)$.

In [13], it was shown that if $T \in B(X)$ has totally finite ascent (in the sense that $T-\lambda$ has finite ascent for each $\lambda \in \mathbb{C}$ ), then Weyl's theorem holds for $T$ if and only if $R(T-\lambda)$ is closed for all $\lambda \in \pi_{00}(T)$. In general, if $T$ has totally finite ascent then $T$ has $\operatorname{SVEP}(15)$. However, the converse is not true. Consider the following example: let $T \in B\left(l_{2}\right)$ be given by

$$
T\left(x_{0}, x_{1}, x_{2}, \cdots\right):=\left(\frac{1}{2} x_{1}, \frac{1}{3} x_{2}, \cdots\right) .
$$


Then clearly $T$ does not have finite ascent. But since $T$ is quasinilpotent, $T$ has SVEP. Thus SVEP is a much weaker condition than having totally finite ascent. However, we can prove:

Theorem 2.2. Suppose that $T \in B(X)$ has SVEP. Then the following statements are equivalent:

(i) Weyl's theorem holds for T;

(ii) $R(T-\lambda)$ is closed for all $\lambda \in \pi_{00}(T)$;

(iii) $\mathcal{X}_{T}(\{\lambda\})$ is finite dimensional for every $\lambda \in \pi_{00}(T)$;

(iv) $\gamma_{T}(\zeta)$ is discontinuous on $\pi_{00}(T)$, where $\gamma_{T}(\cdot)$ denotes the reduced minimum modulus of $T$, i.e.,

$$
\gamma_{T}(\zeta):=\inf \left\{\frac{\|(T-\zeta) x\|}{\operatorname{dist}(x, N(T-\zeta))}: x \in X \backslash N(T-\zeta)\right\}
$$

Proof. (i) $\Rightarrow\left(\right.$ ii): Suppose $\lambda \in \pi_{00}(T)$. Since Weyl's theorem holds for $T$, $\lambda \in \sigma(T) \backslash \omega(T)$. Therefore $R(T-\lambda)$ is closed.

(ii) $\Rightarrow$ (iii): Let $\lambda \in \pi_{00}(T)$. By Lemma 1.2, $T=T_{1} \oplus T_{2}$ on $\mathcal{X}_{T}(\{\lambda\}) \oplus$ $K(T-\lambda)$, where $\sigma\left(T_{1}\right)=\{\lambda\}$ and $\sigma\left(T_{2}\right)=\sigma(T) \backslash\{\lambda\}$. Since $R(T-\lambda)$ is closed, an application of the punctured neighborhood theorem shows that $\lambda$ is a Riesz point of $T$. Therefore $R(P)$ is finite dimensional, where $P \in B(X)$ is the spectral projection corresponding to $\lambda$, given by

$$
P:=\frac{1}{2 \pi i} \int_{\partial D}(z-T)^{-1} d z
$$


where $D$ is an open disk of center $\lambda$ which contains no other points of $\sigma(T)$. Now,

$$
R(P)=\left\{x \in X: \lim _{n \rightarrow \infty}\left\|(T-\lambda)^{n} x\right\|^{\frac{1}{n}}=0\right\}=\mathcal{X}_{T}(\{\lambda\})
$$

hence $\mathcal{X}_{T}(\{\lambda\})$ is finite dimensional.

(iii) $\Rightarrow(\mathrm{i})$ : Suppose $\lambda \in \sigma(T) \backslash \omega(T)$. Then $T-\lambda$ is Weyl but not invertible. We first show that $\lambda \in \partial \sigma(T)$. Assume to the contrary that $\lambda \in \operatorname{int} \sigma(T)$. Then there exists a neighborhood $U$ of $\lambda$ such that $\operatorname{dim} N(T-\mu)>0$ for all $\mu \in U$. It follows from [8, Theorem 10] that $T$ does not have SVEP, a contradiction. Therefore $\lambda \in \partial \sigma(T) \backslash \omega(T)$, and it follows from the punctured neighborhood theorem that $\lambda \in \pi_{00}(T)$. Conversely, suppose that $\lambda \in \pi_{00}(T)$. By Lemma 1.2, $T=T_{1} \oplus T_{2}$ on $\mathcal{X}_{T}(\{\lambda\}) \oplus K(T-\lambda)$, where $\sigma\left(T_{1}\right)=\{\lambda\}$ and $\sigma\left(T_{2}\right)=\sigma(T) \backslash\{\lambda\}$. Since $\mathcal{X}_{T}(\{\lambda\})$ is a finite dimensional subspace of $X, T-\lambda$ is Weyl. Therefore $\lambda \in \sigma(T) \backslash \omega(T)$.

(i) $\Leftrightarrow($ iv): If Weyl's theorem holds for $T$, then it follows from [9, Theorem 1] that $\gamma_{T}(\lambda)$ is discontinuous for each $\lambda \in \pi_{00}(T)$. Conversely, suppose $\gamma_{T}(\lambda)$ is discontinuous on $\pi_{00}(T)$. Since $T$ has SVEP, it suffices to show that $\pi_{00}(T) \subseteq \sigma(T) \backslash \omega(T)$. Suppose that $\lambda \in \pi_{00}(T)$. Since $\lambda$ is an isolated point of $\sigma(T)$, there exist $\delta>0$ and an open disk $D(\lambda, \delta)$ centered in $\lambda$ such that $D(\lambda, \delta) \cap \sigma(T)=\{\lambda\}$. For every $\mu \in D(\lambda, \delta) \backslash\{\lambda\}$, 
we have $T-\mu$ injective and thus,

$$
\begin{aligned}
\gamma_{T}(\mu) & =\inf _{x \in X \backslash N(T-\mu)} \frac{\|(T-\mu) x\|}{\operatorname{dist}(x, N(T-\mu))}=\inf _{x \neq 0} \frac{\|(T-\mu) x\|}{\|x\|} \\
& \leq \inf _{x \in N(T-\lambda) \backslash\{0\}} \frac{\|(T-\lambda) x-(\mu-\lambda) x\|}{\|x\|} \\
& =\inf _{x \in N(T-\lambda) \backslash\{0\}} \frac{\|(\mu-\lambda) x\|}{\|x\|}=|\mu-\lambda| .
\end{aligned}
$$

Since $\gamma_{T}(\zeta)$ is discontinuous at $\lambda, \gamma_{T}(\lambda)>0$. Therefore $R(T-\lambda)$ is closed, and hence $T-\lambda$ is Weyl. This completes the proof.

Before we state our next theorem, we need a definition and two preliminary results. Recall that an operator $T \in B(X)$ is called transaloid if $T-\lambda$ is normaloid for every $\lambda \in \mathbb{C}$.

Lemma 2.3. Suppose that $T \in B(X)$ is transaloid. Then

$$
\mathcal{X}_{T}(\{\lambda\})=N(T-\lambda) \quad \text { for every } \lambda \in \mathbb{C} .
$$

Proof. Observe that $\mathcal{X}_{T}(\{\lambda\})=\left\{x \in X: \lim _{n \rightarrow \infty}\left\|(T-\lambda)^{n} x\right\|^{\frac{1}{n}}=0\right\}$ for each $\lambda \in \mathbb{C}$. Since $T$ is transaloid, $T-\mu$ is normaloid for each $\mu \in \mathbb{C}$. Therefore $\|(T-\lambda) x\| \leq\left\|(T-\lambda)^{n} x\right\|^{\frac{1}{n}}$ for all $x \in X$ and $n \in \mathbb{N}$, and hence $\mathcal{X}_{T}(\{\lambda\}) \subseteq N(T-\lambda)$ for every $\lambda \in \mathbb{C}$. The converse is clear.

Lemma 2.4. Suppose that $T \in B(X)$ has SVEP and is transaloid. Then $T$ is isoloid.

Proof. Suppose that $\lambda$ is an isolated point of $\sigma(T)$. Then it follows from [17 that $X=\mathcal{X}_{T}(\{\lambda\})+X_{T}(\mathbb{C} \backslash\{\lambda\})$. Assume to the contrary that $T-\lambda$ 
is injective. It follows from Lemma 2.3 that $X=X_{T}(\mathbb{C} \backslash\{\lambda\})$. But $(T-\lambda) X_{T}(\mathbb{C} \backslash\{\lambda\})=X_{T}(\mathbb{C} \backslash\{\lambda\})$; hence $T-\lambda$ is surjective. Therefore $T-\lambda$ is invertible, a contradiction. It follows that $T$ is isoloid.

In the following theorem, recall that $H(\sigma(T))$ is the space of functions analytic in an open neighborhood of $\sigma(T)$.

Theorem 2.5. Suppose that $T \in B(X)$ has $S V E P$ and is transaloid. Then Weyl's theorem holds for $f(T)$ for every $f \in H(\sigma(T))$.

Proof. We first show that Weyl's theorem holds for T. Since SVEP and being transaloid are translation-invariant properties, it suffices to show that

$$
0 \in \pi_{00}(T) \Longleftrightarrow T \text { is Weyl and not invertible. }
$$

Suppose $0 \in \pi_{00}(T)$. Then using the spectral projection $P:=\frac{1}{2 \pi i} \int_{\partial D}(\lambda-$ $T)^{-1} d \lambda$, where $D$ is an open disk of center 0 which contains no other points of $\sigma(T)$, we can write $T=T_{1} \oplus T_{2}$, where $\sigma\left(T_{1}\right)=\{0\}$ and $\sigma\left(T_{2}\right)=\sigma(T) \backslash\{0\} . \quad$ It follows from Lemma 2.3 that $P(X)=\{x \in$ $\left.X: \lim _{n \rightarrow \infty}\left\|T^{n} x\right\|^{\frac{1}{n}}=0\right\}=\mathcal{X}_{T}(\{0\})=N(T)$. Since $N(T)$ is a finite dimensional subspace of $X$, we must have $\omega(T)=\omega\left(T_{2}\right)$. But $T_{2}$ is invertible, hence $T$ is Weyl. Therefore $0 \in \sigma(T) \backslash \omega(T)$. Conversely, suppose that $0 \in \sigma(T) \backslash \omega(T)$. Then it follows from Lemma 2.3 that $\mathcal{X}_{T}(\{0\})=N(T)$. Since $\mathcal{X}_{T}(\{0\})$ is a closed invariant subspace for $T, T$ 
can be represented by the following $2 \times 2$ operator matrix:

$$
T=\left(\begin{array}{ll}
0 & T_{1} \\
0 & T_{2}
\end{array}\right) .
$$

Since $\mathcal{X}_{T}(\{0\})$ is a finite dimensional subspace of $X, T$ is Weyl if and only if $T_{2}$ is Weyl. But $\mathcal{X}_{T}(\{0\})=N(T)$; hence $T_{2}$ is injective, and so $T_{2}$ is invertible. It follows from the punctured neighborhood theorem that $0 \in \pi_{00}(T)$. Thus Weyl's theorem holds for $T$.

We now claim that

$$
f(\omega(T))=\omega(f(T)) \text { for all } f \in H(\sigma(T)) .
$$

Let $f \in H(\sigma(T))$. Since $\omega(f(T)) \subseteq f(\omega(T))$ with no restriction on $T$, it suffices to show that $f(\omega(T)) \subseteq \omega(f(T))$. Suppose $\lambda \notin \omega(f(T))$. Then $f(T)-\lambda$ is Weyl and

$$
f(T)-\lambda=c\left(T-\alpha_{1}\right)\left(T-\alpha_{2}\right) \cdots\left(T-\alpha_{n}\right) g(T)
$$

where $c, \alpha_{1}, \alpha_{2}, \cdots, \alpha_{n} \in \mathbb{C}$, and $g(T)$ is invertible. Since the operators on the right-hand side of (2.3) commute, every $T-\alpha_{i}$ is Fredholm. Since $T$ has SVEP, it follows from Lemma 1.1 that each $T-\alpha_{i}$ has finite ascent. Now we show that $i\left(T-\alpha_{i}\right) \leq 0$ for each $i=1,2, \cdots, n$. Observe that if $A \in B(X)$ is Fredholm of finite ascent then $i(A) \leq 0$ : indeed, either $A$ has finite descent, in which case $A$ is Browder and $i(A)=0$, or $A$ has infinite descent and

$$
n \cdot i(A)=\alpha\left(A^{n}\right)-\beta\left(A^{n}\right) \longrightarrow-\infty \quad \text { as } n \longrightarrow \infty,
$$


which implies that $i(A)<0$. Thus $i\left(T-\alpha_{i}\right) \leq 0$ for each $i=1,2, \cdots, n$. Therefore $\lambda \notin f(\omega(T))$, and hence $f(\omega(T))=\omega(f(T))$.

We now recall that if $T$ is isoloid then

$$
f\left(\sigma(T) \backslash \pi_{00}(T)\right)=\sigma(f(T)) \backslash \pi_{00}(f(T))
$$

for every $f \in H(\sigma(T))$ [18, Lemma]. By Lemma 2.4, $T$ is isoloid, and since Weyl's theorem holds for $T$, we have

$$
\begin{aligned}
\sigma(f(T)) \backslash \pi_{00}(f(T)) & =f\left(\sigma(T) \backslash \pi_{00}(T)\right) \quad(\text { by }(2.4) \\
& =f(\omega(T))=\omega(f(T)) \quad(\text { by 2.2 }) .
\end{aligned}
$$

Therefore

$$
\pi_{00}(f(T))=\sigma(f(T)) \backslash\left[\sigma(f(T)) \backslash \pi_{00}(f(T))\right]=\sigma(f(T)) \backslash \omega(f(T)),
$$

so Weyl's theorem holds for $f(T)$, as desired.

From the proof of Theorem 2.5 we obtain the following useful consequence.

Corollary 2.6. Let $T \in B(X)$. Suppose that $T$ or $T^{*}$ has $S V E P$. Then

$$
\omega(f(T))=f(\omega(T)) \quad \text { for every } f \in H(\sigma(T)) .
$$

\section{EXTENSIONS OF $a$-WeYL'S THEOREM}

Let $T \in B(X)$. It is known that the inclusion $\sigma_{e a}(f(T)) \subseteq f\left(\sigma_{e a}(T)\right)$ holds for every $f \in H(\sigma(T))$, with no restriction on $T$ 20]. The next 
theorem shows that the spectral mapping theorem holds for the essential approximate point spectrum, for operators having SVEP.

Theorem 3.1. Let $T \in B(X)$ and suppose that $T$ or $T^{*}$ has $S V E P$. Then

$$
\sigma_{e a}(f(T))=f\left(\sigma_{e a}(T)\right) \quad \text { for every } f \in H(\sigma(T)) .
$$

Proof. Let $f \in H(\sigma(T))$. It suffices to show that $f\left(\sigma_{e a}(T)\right) \subseteq \sigma_{e a}(f(T))$. Suppose that $\lambda \notin \sigma_{e a}(f(T))$. Then $f(T)-\lambda \in \Phi_{+}^{-}(X)$ and

$$
f(T)-\lambda=c\left(T-\alpha_{1}\right)\left(T-\alpha_{2}\right) \cdots\left(T-\alpha_{n}\right) g(T)
$$

where $c, \alpha_{1}, \alpha_{2}, \cdots, \alpha_{n} \in \mathbb{C}$, and $g(T)$ is invertible. Since the operators on the right-hand side of (3.1) commute, $T-\alpha_{i} \in \Phi_{+}(X)$. Since $T$ has SVEP, it follows from Lemma 1.1 that each $T-\alpha_{i}$ has finite ascent. Therefore by the proof of Theorem 2.5, $i\left(T-\alpha_{i}\right) \leq 0$ for each $i=$ $1,2, \cdots, n$. It follows that $\lambda \notin f\left(\sigma_{e a}(T)\right)$.

Suppose now that $T^{*}$ has SVEP. Since $T-\alpha_{i} \in \Phi_{+}(X), T^{*}-\alpha_{i} \in$ $\Phi_{-}\left(X^{*}\right)$. Since $T^{*}$ has SVEP, it follows from Lemma 1.1 that each $T-\alpha_{i}$ has finite descent. We claim that $i\left(T-\alpha_{i}\right) \geq 0$ for each $i=$ $1,2, \cdots, n$. Observe that if $A \in \Phi_{-}(X)$ and $A$ is not Fredholm then evidently $i(A) \geq 0$. If $A$ is Fredholm with finite descent, then either $A$ has finite ascent (and then $A$ is Browder and $i(A)=0$ ), or $A$ has infinite ascent (and then

$$
n \cdot i(A)=\alpha\left(A^{n}\right)-\beta\left(A^{n}\right) \longrightarrow \infty \quad \text { as } n \longrightarrow \infty,
$$


which implies that $i(A)>0)$. Thus $i\left(T-\alpha_{i}\right) \geq 0$ for each $i=1,2, \cdots, n$. However,

$$
0 \leq \sum_{i=1}^{n} i\left(T-\alpha_{i}\right)=i(f(T)-\lambda) \leq 0
$$

and so $T-\alpha_{i}$ is Weyl for each $i=1,2, \cdots, n$. Hence $\lambda \notin f\left(\sigma_{e a}(T)\right)$, and so $\sigma_{e a}(f(T))=f\left(\sigma_{e a}(T)\right)$. This completes the proof of the theorem.

In general, we cannot expect that $a$-Weyl's theorem necessarily holds for operators having SVEP. Consider the quasinilpotent operator $T$ on $\ell_{2}$ given by (2.1); this operator has SVEP. But $\sigma_{a}(T)=\sigma_{e a}(T)=\{0\}$, and $\pi_{00}^{a}(T)=\{0\}$; hence $a$-Weyl's theorem does not hold for $T$. However, $a$-Browder's theorem does hold, as the following result shows.

Theorem 3.2. Suppose that $T \in B(X)$ has $S V E P$ and $S \in B(X)$ satisfies $S \prec T$. Then a-Browder's theorem holds for $f(S)$, for every $f \in H(\sigma(S))$.

Proof. We first recall that $S$ has SVEP. For, let $U$ be any open set and $f: U \longrightarrow X$ be any analytic function such that $(S-\lambda) f(\lambda)=0$ for all $\lambda \in U$. Since $S \prec T$, there exists a quasiaffinity $A$ such that $A S=T A$. Thus, $A(S-\lambda)=(T-\lambda) A$ for all $\lambda \in U$. Since $(S-\lambda) f(\lambda)=0$ for all $\lambda \in U, 0=A(S-\lambda) f(\lambda)=(T-\lambda) A f(\lambda)$ for all $\lambda \in U$. But $T$ has SVEP, hence $A f(\lambda)=0$ for all $\lambda \in U$. Since $A$ is a quasiaffinity, $f(\lambda)=0$ for all $\lambda \in U$. Therefore $S$ has SVEP. Next we show that $a$-Browder's theorem holds for $S$, i.e., that $\sigma_{e a}(S)=\sigma_{a b}(S)$. It is well known that 
$\sigma_{e a}(S) \subseteq \sigma_{a b}(S)$. To prove the converse, suppose that $\lambda \in \sigma_{a}(S) \backslash \sigma_{e a}(S)$. Then $S-\lambda \in \Phi_{+}^{-}(X)$ and $S-\lambda$ is not bounded below. Since $S$ has SVEP and $S-\lambda \in \Phi_{+}^{-}(X)$, it follows from Lemma 1.1 that $S-\lambda$ has finite ascent. Therefore by [20, Theorem 2.1], $\lambda \in \sigma_{a}(S) \backslash \sigma_{a b}(S)$. Thus $a$-Browder's theorem holds for $S$. Hence, it follows from Theorem 3.1 that

$$
\sigma_{a b}(f(S))=f\left(\sigma_{a b}(S)\right)=f\left(\sigma_{e a}(S)\right)=\sigma_{e a}(f(S))
$$

(all $f \in H(\sigma(S)))$, and so $a$-Browder's theorem holds for $f(S)$.

In analogy with Theorem 2.2, we obtain

Corollary 3.3. Suppose that $T \in B(X)$ has $S V E P$ and $S \in B(X)$ satisfies $S \prec T$. The following statements are equivalent:

(i) a-Weyl's theorem holds for $S$;

(ii) $R(S-\lambda)$ is closed for all $\lambda \in \pi_{00}^{a}(S)$;

(iii) $\gamma_{S}(\zeta)$ is discontinuous on $\pi_{00}^{a}(S)$, where $\gamma_{S}(\cdot)$ denotes the reduced minimum modulus;

(iv) $\sigma_{e a}(S) \cap \pi_{00}^{a}(S)=\emptyset$;

(v) $\pi_{00}^{a}(S)=\sigma_{a}(S) \backslash \sigma_{a b}(S)$.

Proof. Since $T$ has SVEP and $S \prec T$, it follows from Theorem 3.2 that $a$-Browder's theorem holds for $S$. Therefore $\sigma_{e a}(S)=\sigma_{a b}(S)$. (i) $\Leftrightarrow\left(\right.$ ii): Suppose $\lambda \in \pi_{00}^{a}(S)$. Since $a$-Weyl's theorem holds for $S$, $\lambda \in \sigma_{a}(S) \backslash \sigma_{e a}(S)$. Therefore $R(S-\lambda)$ is closed. Conversely, suppose that $R(S-\lambda)$ is closed for all $\lambda \in \pi_{00}^{a}(S)$. Since $a$-Browder's theorem 
holds for $S, \sigma_{e a}(S)=\sigma_{a b}(S)$. It follows from [20, Corollary 2.2] that $\sigma_{a}(S) \backslash \sigma_{e a}(S)=\sigma_{a}(S) \backslash \sigma_{a b}(S) \subseteq \pi_{00}^{a}(S)$. Conversely, let $\lambda \in \pi_{00}^{a}(S)$. Then $S-\lambda$ has closed range, and so $S-\lambda \in \Phi_{+}(X)$. Since $S$ has SVEP, it follows from Lemma 1.1 that $S-\lambda$ has finite ascent. Therefore $i(S-\lambda) \leq 0$, and hence $S-\lambda \in \Phi_{+}^{-}(X)$.

(i) $\Leftrightarrow$ (iii): If $a$-Weyl's theorem holds for $S$, then it follows from 19, Theorem 2.4] that $\gamma_{S}(\lambda)$ is discontinuous for each $\lambda \in \pi_{00}^{a}(S)$. Conversely, suppose that $\gamma_{S}(\lambda)$ is discontinuous on $\pi_{00}^{a}(S)$. To show that $a$-Weyl's theorem holds for $S$, it suffices to show that $R(S-\lambda)$ is closed for all $\lambda \in \pi_{00}^{a}(S)$. Let $\lambda \in \pi_{00}^{a}(S)$. Since $\lambda$ is an isolated point of $\sigma_{a}(S)$, there exist $\epsilon>0$ and an open disk $D(\lambda, \epsilon)$ centered in $\lambda$ such that $D(\lambda, \epsilon) \cap \sigma_{a}(S)=\{\lambda\}$. For every $\mu \in D(\lambda, \epsilon) \backslash\{\lambda\}$, we have $S-\mu$ injective, and therefore

$$
\begin{aligned}
\gamma_{S}(\mu) & =\inf _{x \in X \backslash N(S-\mu)} \frac{\|(S-\mu) x\|}{\operatorname{dist}(x, N(S-\mu))}=\inf _{x \neq 0} \frac{\|(S-\mu) x\|}{\|x\|} \\
& \leq \inf _{x \in N(S-\lambda) \backslash\{0\}} \frac{\|(S-\lambda) x-(\mu-\lambda) x\|}{\|x\|} \\
& =\inf _{x \in N(S-\lambda) \backslash\{0\}} \frac{\|(\mu-\lambda) x\|}{\|x\|}=|\mu-\lambda| .
\end{aligned}
$$

Since $\gamma_{S}(\zeta)$ is discontinuous at $\lambda, \gamma_{S}(\lambda)>0$. It follows that $R(S-\lambda)$ is closed.

(ii) $\Leftrightarrow\left(\right.$ iv): Assume to the contrary that $\lambda \in \sigma_{e a}(S) \cap \pi_{00}^{a}(S)$, so $R(S-\lambda)$ is closed. Since $S$ has SVEP, $i(S-\lambda) \leq 0$. Therefore $S-\lambda \in \Phi_{+}^{-}(X)$, and so $\lambda \notin \sigma_{e a}(S)$, a contradiction. Conversely, let $\lambda \in \pi_{00}^{a}(S)$. Since 
$\sigma_{e a}(S) \cap \pi_{00}^{a}(S)=\emptyset, \lambda \notin \sigma_{e a}(S)$. Therefore $R(S-\lambda)$ is closed.

$(\mathrm{i}) \Leftrightarrow(\mathrm{v})$ : Since $a$-Weyl's theorem holds for $S$, we have

$$
\pi_{00}^{a}(S)=\sigma_{a}(S) \backslash \sigma_{e a}(S)=\sigma_{a}(S) \backslash \sigma_{a b}(S)
$$

Conversely, suppose that $\pi_{00}^{a}(S)=\sigma_{a}(S) \backslash \sigma_{a b}(S)$. Since $S$ has SVEP, $\sigma_{e a}(S)=\sigma_{a b}(S)$. Therefore $\pi_{00}^{a}(S)=\sigma_{a}(S) \backslash \sigma_{e a}(S)$, and hence $a$-Weyl's theorem holds for $S$. This completes the proof.

Recall [12, Definition 13] that an operator $T \in B(X)$ is called reguloid if each isolated point of its spectrum is a regular point, in the sense that there is a generalized inverse $S_{\lambda} \in B(X)$, i.e., $(T-\lambda)=(T-\lambda) S_{\lambda}(T-\lambda)$.

Corollary 3.4. Let $T \in B(X)$. Suppose that $T$ or $T^{*}$ has Dunford's property $(C)$ and $T$ is reguloid. Then Weyl's theorem holds for $f(T)$ for every $f \in H(\sigma(T))$.

Proof. Since Dunford's property (C) implies SVEP [17, Proposition 1.2.19], it follows from Theorem 3.2 that Browder's theorem holds for T. But $T$ is reguloid, hence $T-\lambda$ has closed range for each $\lambda \in \pi_{00}(T)$. Therefore Weyl's theorem holds for $T$ by Theorem 2.2. Since $T$ is reguloid, it is also isoloid by [12, Theorem 14]. Hence by the proof of Theorem 2.5, Weyl's theorem holds for $f(T)$, for each $f \in H(\sigma(T))$.

Finally, recall that $T \in B(X)$ is called approximate isoloid ( $a$-isoloid) if every isolated point of $\sigma_{a}(T)$ is an eigenvalue of $T$. We observe that if $T$ is $a$-isoloid then it is isoloid because the boundary of $\sigma(T)$ is contained 
in $\sigma_{a}(T)$. (However, the converse is not true. Consider the following example: let $T=T_{1} \oplus T_{2}$, where $T_{1}$ is the unilateral shift on $l_{2}$ and $T_{2}$ is injective and quasinilpotent on $l_{2}$. Then $\sigma(T)=\{z \in C:|z| \leq 1\}$ and $\sigma_{a}(T)=\{z \in C:|z|=1\} \cup\{0\}$. Therefore $T$ is isoloid but not $a$-isoloid.)

Theorem 3.5. Suppose that $T^{*} \in B\left(X^{*}\right)$ has SVEP and $T$ is transaloid. Then a-Weyl's theorem holds for $T$. If, in addition, $T$ is a-isoloid, then a-Weyl's theorem holds for $f(T)$, for every $f \in H(\sigma(T))$.

Proof. We first show that $a$-Weyl's theorem holds for $T$. Since SVEP and being transaloid are translation-invariant properties, it suffices to show that

$$
0 \in \pi_{00}^{a}(T) \Longleftrightarrow 0 \in \sigma_{a}(T) \backslash \sigma_{e a}(T)
$$

Suppose that $0 \in \pi_{00}^{a}(T)$. Since $T^{*}$ has SVEP, it follows from that $[8$, Corollary 7] that $\sigma(T)=\sigma_{a}(T)$. Therefore 0 is an isolated point of $\sigma(T)$. Using the spectral projection $P:=\frac{1}{2 \pi i} \int_{\partial D}(\lambda-T)^{-1} d \lambda$, where $D$ is an open disk of center 0 which contains no other points of $\sigma(T)$, we can represent $T$ as the direct sum

$$
T=\left(\begin{array}{cc}
T_{1} & 0 \\
0 & T_{2}
\end{array}\right)
$$


where $\sigma\left(T_{1}\right)=\{0\}$ and $\sigma\left(T_{2}\right)=\sigma(T) \backslash\{0\}$. It follows from Lemma 2.3 that

$$
P(X)=\left\{x \in X: \lim _{n \rightarrow \infty}\left\|T^{n} x\right\|^{\frac{1}{n}}=0\right\}=\mathcal{X}_{T}(\{0\})=N(T) .
$$

Since $N(T)$ is a finite dimensional subspace of $X, \omega(T)=\omega\left(T_{2}\right)$. But $T_{2}$ is invertible, hence $T$ is Weyl. Therefore $0 \in \sigma_{a}(T) \backslash \sigma_{e a}(T)$. Conversely, suppose that $0 \in \sigma_{a}(T) \backslash \sigma_{e a}(T)$. Then $T \in \Phi_{+}(X)$ and $i(T) \leq 0$. Since $T^{*}$ has SVEP, it follows from Lemma 1.1 that $i(T) \geq 0$. Therefore $T$ is Weyl, and so $0 \in \sigma_{a}(T) \backslash \omega(T)$. Observe now that Browder's theorem holds for $T^{*}$ by Theorem 3.2. Also, Browder's theorem holds for $T^{*}$ if and only if it holds for $T$. Thus Browder's theorem holds for $T$, and so $0 \in \pi_{00}^{a}(T)$.

We have thus established that $a$-Weyl's theorem holds for $T$. Let $f \in H(\sigma(T))$. Since $T^{*}$ has SVEP, it follows from Theorem 3.1 that $\sigma_{e a}(f(T))=f\left(\sigma_{e a}(T)\right)$. But $a$-Weyl's theorem holds for $T$, hence $\sigma_{e a}(T)=\sigma_{a b}(T)$. Therefore

$$
\sigma_{e a}(f(T))=f\left(\sigma_{e a}(T)\right)=f\left(\sigma_{a b}(T)\right)=\sigma_{a b}(f(T))
$$

(the last equality by virtue of [20, Theorem 3.4]) and so $a$-Browder's theorem holds for $f(T)$. Now let $\lambda \in \pi_{00}^{a}(f(T))$. Since $\sigma(T)=\sigma_{a}(T)$, it follows that $\sigma\left(f(T)=\sigma_{a}(f(T))\right.$, so $\lambda \in \pi_{00}(f(T))$. Since $T$ is $a$-isoloid, we notice that Weyl's theorem holds for $f(T)$ by the proof of Theorem 2.5. Therefore $f(T)-\lambda$ has closed range for all $\lambda \in \pi_{00}^{a}(f(T))$, and it follows from Corollary 3.3 that $a$-Weyl's theorem holds for $f(T)$. 
[1] P. Aiena and O. Monsalve, Operators which do not have the single valued extension property, J. Math. Anal. Appl. 250 (2000), 435-448.

[2] S.K. Berberian, An extension of Weyl's theorem to a class of not necessarily normal operators, Michigan Math. J. 16 (1969), 273-279.

[3] S.K. Berberian, The Weyl spectrum of an operator, Indiana Univ. Math. J. 20 (1970), 529-544.

[4] L.A. Coburn, Weyl's theorem for nonnormal operators, Michigan Math. J. 13 (1966), 285-288.

[5] I. Colojoara and C. Foias, Theory of Generalized Spectral Operators, Gordon and Breach, New York, 1968.

[6] S.V. Djordjević and D.S. Djordjević, Weyl's theorems: continuity of the spectrum and quasihyponormal operators, Acta Sci. Math. (Szeged) 64 (1998), 259-269.

[7] S.V. Djordjević and Y.M. Han, Browder's theorems and spectral continuity, Glasgow Math. J. 42 (2000), 479-486.

[8] J.K. Finch, The single valued extension property on a Banach space, Pacific J. Math. 58 (1975), 61-69.

[9] K. Gustafson, Necessary and sufficient conditions for Weyl's theorem, Michigan Math. J. 19 (1972), 71-81.

[10] R.E. Harte, Fredholm, Weyl and Browder theory, Proc. Royal Irish Acad. 85A (1985), 151-176.

[11] R.E. Harte, Invertibility and Singularity for Bounded Linear Operators, Dekker, New York, 1988.

[12] R.E. Harte and W.Y. Lee, Another note on Weyl's theorem, Trans. Amer. Math. Soc. 349 (1997), 2115-2124.

[13] I.H. Jeon, Weyl's theorem and quasi-similarity, Integral Equations Operator Theory 39 (2001), 214-221. 
[14] J.J. Koliha, Isolated spectral points, Proc. Amer. Math. Soc. 124 (1996), 34173424.

[15] K.B. Laursen, Operators with finite ascent, Pacific J. Math. 152 (1992), 323-336.

[16] K.B. Laursen, Essential spectra through local spectral theory, Proc. Amer. Math. Soc. 125 (1997), 1425-1434.

[17] K.B. Laursen and M.M. Neumann, An Introduction to Local Spectral Theory, London Mathematical Society Monographs New Series 20, Clarendon Press, Oxford, 2000.

[18] S.H. Lee and W.Y. Lee, A spectral mapping theorem for the Weyl spectrum, Glasgow Math. J. 38 (1996), 61-64.

[19] V. Rakočević, On the essential approximate point spectrum II, Mat. Vesnik. 36 (1984), 89-97.

[20] V. Rakočević, Approximate point spectrum and commuting compact perturbations, Glasgow Math. J. 28 (1986), 193-198.

[21] H. Weyl, Über beschränkte quadratische Formen, deren Differenz vollsteig ist, Rend. Circ. Mat. Palermo 27 (1909), 373-392.

Department of Mathematics, The University of Iowa, Iowa City, Iowa 52242

E-mail address: curto@math.uiowa.edu

Department of Mathematics, The University of Iowa, Iowa City, Iowa 52242

E-mail address: yhan@math.uiowa.edu 